\title{
Linoleic Acid Attenuates Endothelium-Derived Relaxing Factor Production by Suppressing cAMP-Hydrolyzing Phosphodiesterase Activity
}

\author{
Jiazhang Wei, MD; Kazuhiko Takeuchi, MD, PhD; Hiroshi Watanabe, MD, PhD
}

\begin{abstract}
Background: Linoleic acid (LA) promotes monocyte chemotaxis and cell adhesion molecules such as MCP-1 and VCAM-1, which contribute to atherosclerogenesis. These molecules are restrained by endothelium-derived relaxing factors (EDRFs), such as nitric oxide (NO) and prostaglandin $\mathrm{I}_{2}\left(\mathrm{PGI}_{2}\right)$. Hence, the expressions of MCP-1 and VCAM1 upregulated by LA may be partly attributable to decreased EDRF production. However, effect of LA on EDRF production remains controversial.
\end{abstract}

\begin{abstract}
Methods and Results: The present study aimed to examine the effects of LA and other free fatty acids on EDRF production and the endothelial $\mathrm{Ca}^{2+}$ responses that mediate EDRF production, using primary cultured porcine aortic endothelial cells (PAECs). LA at $0.1-5 \mu \mathrm{mol} / \mathrm{L}$ attenuated bradykinin (BK)-induced $\mathrm{NO}$ and $\mathrm{PGI}_{2}$ production while suppressing the $\mathrm{BK}$-induced $\mathrm{Ca}^{2+}$ response dose-dependently. The inhibitory effect of $\mathrm{LA}$ on the $\mathrm{Ca}^{2+}$ response was eliminated by adenylate cyclase inhibitor SQ22536, boosted by cAMP-hydrolyzing phosphodiesterase (PDE) inhibitor, rolipram, and mimicked by plasma membrane permeable 8-bromo-cAMP. Moreover, LA was confirmed to dose-dependently increase intracellular cAMP levels and selectively inhibit cAMP-hydrolyzing PDE activity in vitro. In contrast, none of palmitic, stearic, or oleic acid affected BK-induced EDRF production or $\mathrm{Ca}^{2+}$ responses, or induced intracellular cAMP accumulation.
\end{abstract}

Conclusions: LA induced intracellular cAMP accumulation by inhibiting cAMP-hydrolyzing PDE activity, thus resulting in attenuation of $\mathrm{Ca}^{2+}$ responses and EDRF production in PAECs. (Circ $J$ 2013; 77: 2823-2830)

Key Words: Cyclic AMP; Endothelial cells; Endothelium-derived relaxing factor; Linoleic acid; Phosphdiesterase

$\mathbf{E}$ levated plasma concentration of free fatty acids (FFAs) has been found to be associated with an increased risk of developing symptomatic ischemic heart disease (IHD). ${ }^{1}$ Moreover, in a subanalysis of the relationship between individual FFAs and the risk of major coronary events in the prospective randomized clinical trial, JELIS (the Japan EPA Lipid Intervention Study), only a high concentration of linoleic acid (LA) was found to be an independent risk factor of major coronary events. ${ }^{2}$ Recent, updated data from the Sydney Diet Heart Study revealed that substituting dietary LA for saturated fats increased the rates of death from all causes, coronary artery disease, and cardiovascular disease. ${ }^{3}$ It was demonstrated that LA promoted monocyte chemotaxis and adhesion to endothelial cells (ECs) and enhanced the expression of cell adhesion molecules, such as monocyte chemotactic protein 1 (MCP-1) and vascular cell adhesion molecule 1 (VCAM-1), that contribute to atherosclerogenesis. ${ }^{4,5}$ The expression of MCP-1 and VCAM-1 is restrained by endothelium-derived relaxing factors (EDRFs), such as nitric oxide (NO) and prostaglandin $\mathrm{I}_{2}$
(PGI 2$)^{6-8}$ Hence, enhanced expression of both MCP-1 and VCAM-1 by LA may be partly attributable to a decreased EDRF production. However, the effect of LA on EDRF production remains controversial..$^{910}$ Furthermore, the mechanism by which LA affects EDRF production still needs to be clarified.

\section{Editorial p 2702}

The present study used primary cultured porcine aortic endothelial cells (PAECs) to examine the effects of LA and other individual FFA on EDRF production and the endothelial $\mathrm{Ca}^{2+}$ responses that mediate it. Oleic, palmitic, linoleic, and stearic acids were used for this study because they account for $32 \%$, $27 \%, 26 \%$, and $8 \%$, respectively, of human plasma FFAs. ${ }^{11}$

\section{Methods}

\section{Cell Culture}

Porcine aortas were collected from a local slaughterhouse and

Received February 19, 2013; revised manuscript received May 14, 2013; accepted June 17, 2013; released online July 25, 2013 Time for primary review: 5 days

Department of Clinical Pharmacology and Therapeutics, Hamamatsu University School of Medicine, Hamamatsu, Japan

Mailing address: Kazuhiko Takeuchi, MD, PhD, Department of Clinical Pharmacology and Therapeutics, Hamamatsu University School of Medicine, 1-20-1 Handayama, Higashi-ku, Hamamatsu 431-3192, Japan. E-mail: takeutik@ @ama-med.ac.jp

ISSN-1346-9843 doi:10.1253/circj.CJ-13-0248

All rights are reserved to the Japanese Circulation Society. For permissions, please e-mail: cj@j-circ.or.jp 
the ECs isolated, as previously described, ${ }^{12}$ by gently scraping the intima of the descending portion of each aorta. Human umbilical vein endothelial cells (HUVECs) were provided by Lonza Japan Ltd (Tokyo, Japan). The M199 solution (Boehringer Mannheim, Penzberg, DE) used for cell culture contained $150 \mathrm{mmol} / \mathrm{L} \mathrm{NaCl}, 2.7 \mathrm{mmol} / \mathrm{L} \mathrm{KCl}, 1.2 \mathrm{mmol} / \mathrm{L}$ $\mathrm{MgSO}_{4}, 1.2 \mathrm{mmol} / \mathrm{L} \mathrm{KH}_{2} \mathrm{PO}_{4}, 10 \mathrm{mmol} / \mathrm{L} \mathrm{HEPES}$ and $1 \mathrm{mmol} / \mathrm{L}$ $\mathrm{CaCl}_{2}$. After centrifugation at $250 \mathrm{~g}$ for $10 \mathrm{~min}$ in M199 solution, the sedimented cell fraction was collected and resuspended in M199 solution supplemented with $100 \mathrm{IU} / \mathrm{ml}$ penicillin $\mathrm{G}, 100 \mu \mathrm{g} / \mathrm{ml}$ streptomycin (Sigma-Aldrich, St. Louis, MO, USA), and $20 \%$ newborn calf serum (NCS, Invitrogen, Carlsbad, CA, USA), seeded onto dishes, and cultured in an incubator at $37^{\circ} \mathrm{C}$ under $5 \% \mathrm{CO}_{2}$, with the medium renewed daily. The study conformed to the Guide for the Care and Use of Laboratory Animals published by the US National Institutes of Health (NIH publication, 8th edition, 2010). All experiments were performed in accordance with the regulations of the Animal Research Committee of Hamamatsu University School of Medicine.

\section{Reagents and Solution}

Linoleic, oleic, palmitic, and stearic acids, as well as bradykinin (BK), thapsigargin, 8-bromoadenosine-3', 5' -cyclic monophosphate (8-bromo-cAMP), adenylate cyclase inhibitor, SQ22536, and selective phosphodiesterase type IV (PDE4) inhibitor, rolipram, were purchased from Sigma-Aldrich. Oleic, palmitic, linoleic, and stearic acids were dissolved in DMSO at $10 \mathrm{mmol} / \mathrm{L}$ as stock solutions, which were further dissolved in a modified Tyrode's solution containing $150 \mathrm{mmol} / \mathrm{L} \mathrm{NaCl}$, $2.7 \mathrm{mmol} / \mathrm{L} \mathrm{KCl}, 1.2 \mathrm{mmol} / \mathrm{L} \mathrm{MgSO}_{4}, 1.2 \mathrm{mmol} / \mathrm{L} \mathrm{KH}_{2} \mathrm{PO}_{4}$, $1 \mathrm{mmol} / \mathrm{L} \mathrm{CaCl} 2$ and $10.0 \mathrm{mmol} / \mathrm{L} N$-2-hydroxyethylpiperazine- $N$ '-2-ethanesulfonic acid, with $\mathrm{pH} 7.4$ at $25^{\circ} \mathrm{C}$.

\section{Measurement of Endothelial NO Production}

NO production in the PAECs was measured using a cell permeable, photo-stable, fluorescent dye for NO, 4-amino-5-methylamino-2', 7'-difluorofluorescein diacetate (DAF-FM/DA, Daiichi Pure Chemicals, Tokyo, Japan), which emits increased fluorescence after reaction with an active intermediate of NO formed during spontaneous $\mathrm{NO}$ oxidation to $\mathrm{NO}_{2}{ }^{-}{ }^{13}$ After 2 days of culture, PAECs adhering to glass cover slips were incubated in a modified Tyrode's solution containing $5 \mu \mathrm{mol} / \mathrm{L}$ of DAF-FM/DA for $20 \mathrm{~min}$. The cells were subsequently washed 3 times with the modified Tyrode's solution to remove the DAF-FM/DA from the extracellular fluid and then incubated at room temperature for $75 \mathrm{~min}$ in the modified Tyrode's solution containing various concentrations of linoleic, palmitic, stearic, or oleic acid before BK $(1 \mu \mathrm{mol} / \mathrm{L})$ administration to induce NO production in the PAECs. DAF-FM was excited with 490-nm light and its fluorescence emission intensities measured simultaneously at $510 \mathrm{~nm}$. Fluorescent images were acquired and quantified every $6 \mathrm{~s}$ from individual cells with a fluorescence imaging and analysis system (Aqua Cosmos 2.5, Hamamatsu Photonics) using an ultrahigh-sensitivity television camera (CCD). Changes in DAF-FM fluorescence intensities $(F)$ in each experiment were normalized to the fluorescence intensity recorded at the experiment beginning $\left(\mathrm{F}_{0}\right)$.

\section{Measurement of Endothelial PGI2 Production}

$\mathrm{PGI}_{2}$ released from PAECs was measured as the concentration of its stable metabolite, 6-keto-PGF $1 \alpha$, using an enzyme immunoassay, as described previously. ${ }^{14}$ PAECs were plated in 12-well culture dishes and grown to confluence in M199 medium at $37^{\circ} \mathrm{C}$ under $5 \% \mathrm{CO}_{2}$ and saturated humidity. PAECs were then preincubated in various FFAs as described earlier, and the cell medium was then measured 14 min after BK administration. The 6-keto- $\mathrm{PGF}_{1} \alpha$ concentrations in the cell medium were determined according to the manufacturer's protocol (6-keto-PGF1 $\alpha$ EIA kit, Assay Designs, Ann Arbor, MI, USA). The $\mathrm{pH}$ of the samples was adjusted to 7.4 before 6 -keto-PGF $\alpha$ measurement to eliminate any effects on the immunoassay.

\section{Measurement of Intracellular $\mathrm{Ca}^{2+}$ Concentration in ECs}

Using the acetoxymethyl ester of fura-2 (fura-2/AM, Dojindo Molecular Technologies, Kumamoto, Japan), intracellular $\mathrm{Ca}^{2+}$ concentrations $\left(\left[\mathrm{Ca}^{2+}\right]_{i}\right)$ in individual PAECs were measured as described previously. ${ }^{12}$ After 2 days of culture, PAECs adhering to glass cover slips were incubated for $40 \mathrm{~min}$ in a modified Tyrode's solution containing $10 \% \mathrm{NCS}$ and $2 \mu \mathrm{mol} / \mathrm{L}$ fura-2/AM, a fluorescent $\mathrm{Ca}^{2+}$ indicator. The cells were subsequently washed 3 times with the modified Tyrode's solution to remove the fura-2/AM and the serum from the extracellular fluid, then left to equilibrate in the cell buffer for $20 \mathrm{~min}$ before measurements were started. All experiments were performed at $25^{\circ} \mathrm{C}$. The absorption shift of fura- 2 that occurs upon binding can be determined by scanning the excitation spectrum between 340 and $380 \mathrm{~nm}$ while monitoring the emission at $510 \mathrm{~nm}$. Fluorescent images were acquired and quantified every 30 s from individual cells with a fluorescence imaging and analysis system (Aqua Cosmos 2.5, Hamamatsu Photonics) using an ultrahigh-sensitivity television camera (CCD). After background subtraction, the fluorescence ratio (F340/ F380) was obtained by dividing, pixel by pixel, the $340-\mathrm{nm}$ image by the $380-\mathrm{nm}$ image. Changes in this ratio were used to express changes in the intracellular $\mathrm{Ca}^{2+}$ concentration to eliminate potential artifacts caused by variations in cell thickness, intracellular dye distribution or photobleaching. The minimum and maximum F340/F380 ratios, determined using a Calcium Calibration Buffer Kit (Invitrogen), were 0.46 with no $\mathrm{Ca}^{2+}$ and 27.5 at $39 \mu \mathrm{mol} / \mathrm{L} \mathrm{Ca}^{2+}$.

\section{Measurement of Endothelial cAMP Production}

PAECs were plated in 96-well culture dishes and incubated to confluence with $\mathrm{M} 199$ medium at $37^{\circ} \mathrm{C}$ under $5 \% \mathrm{CO}_{2}$ and saturated humidity. For the experiment, the medium was removed and the cells were washed with a modified Tyrode's solution and then incubated for $75 \mathrm{~min}$ in the modified Tyrode's solution containing various concentrations of FFAs in the presence and absence of $200 \mu \mathrm{mol} / \mathrm{L}$ of SQ22536. Intracellular cAMP concentrations in the PAECs were determined using an enzyme immunoassay according to the manufacturer's protocol (Cyclic AMP EIA Kit, Assay Designs).

\section{Measurement of PDE Activity}

cAMP/cGMP-hydrolyzing PDE activity was determined using a cyclic nucleotide PDE assay kit according to the manufacturer's protocol (Assay Designs). cAMP and cGMP-hydrolyzing PDE activities were evaluated as the cleavage of cAMP and cGMP through cAMP/cGMP-hydrolyzing PDE in $30 \mathrm{~min}$, respectively. The 5'-nucleotide released was further cleaved into the nucleoside and phosphate by the enzyme 5'-nucleotidase. The phosphate released by enzymatic cleavage was quantified using BIOMOL GREEN ${ }^{\mathrm{TM}}$ reagent. A nonspecific cyclic nucleotide PDE inhibitor, 3-isobutyl-1-methylxanthine (IBMX) was included as a test control.

\section{Statistical Analysis}

Statistical analysis was performed by 1-way ANOVA followed 
A

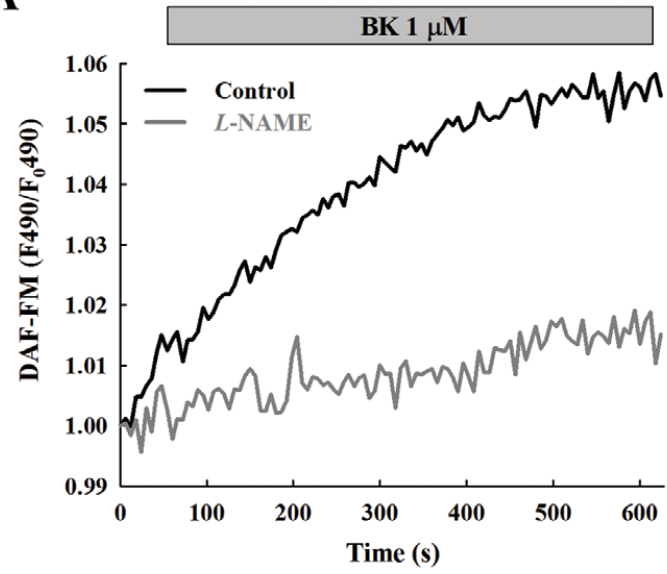

B

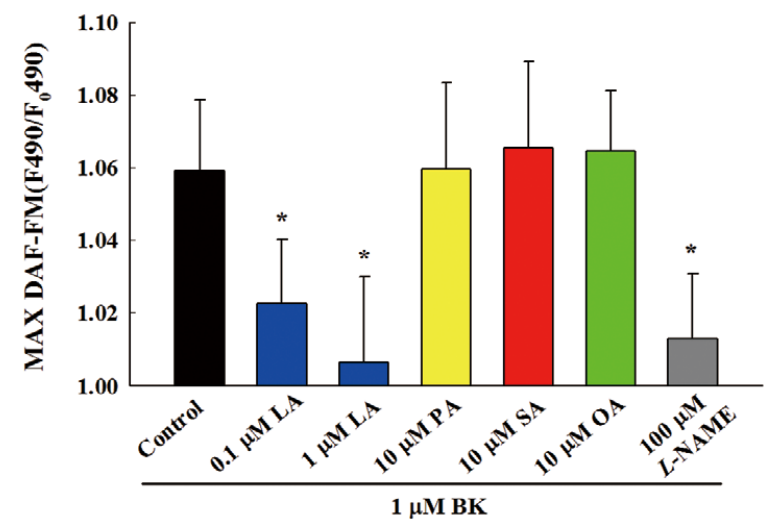

Figure 1. Effects of free fatty acids (FFAs) on bradykinin (BK)-induced nitric oxide (NO) production in porcine aortic endothelial cells (PAECS). (A) Representative traces showing the changes in BK-induced NO production indicated by the F490/F0490 DAF-FM ratio in PAECs pretreated without (control) and with the NO synthase inhibitor, L-NAME (100 $\mu \mathrm{mol} / \mathrm{L})$. (B) Summary of the effects of FFAs on BK-induced NO production. PAECs were pretreated with linoleic (LA), palmitic (PA), stearic (SA), or oleic acid (OA) for $75 \mathrm{~min}$ at the indicated concentrations. Intracellular NO production stimulated by BK is indicated by the DAF-FM F490/Fo490 ratio. The data are the mean $\pm S D$ of 4 independent observations in separate cell culture wells. ${ }^{*} P<0.05$ vs. control.

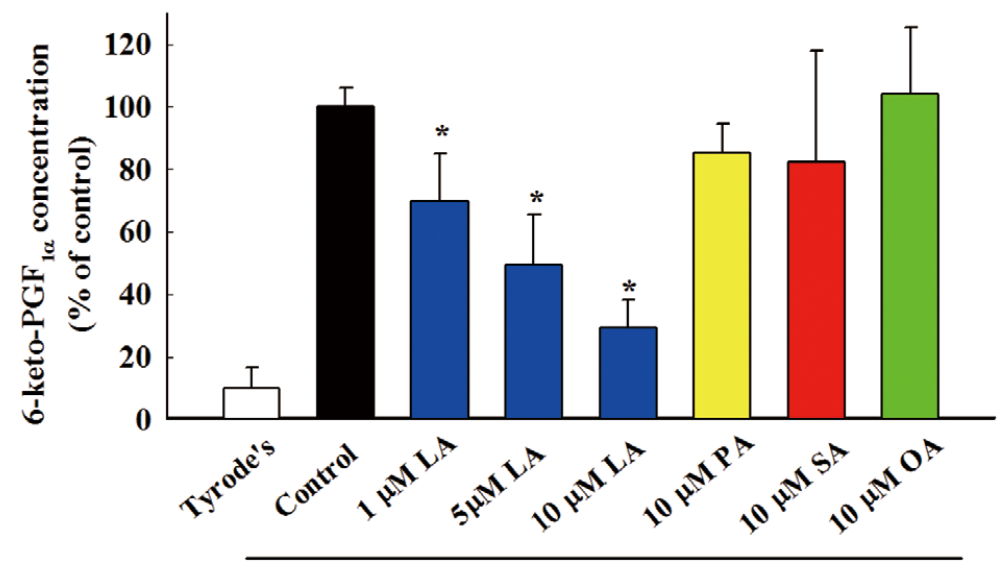

$10 \mathrm{nM}$ BK

Figure 2. Effects of free fatty acids (FFAs) on bradykinin (BK)-induced prostaglandin $\mathrm{I}_{2}\left(\mathrm{PGI}_{2}\right)$ production in porcine aortic endothelial cells (PAECs) pretreated with FFAs for 75 min at the indicated concentrations. BK-induced PGI 2 production in PAECs was estimated as the concentration of 6-keto-PGF1a by enzyme immunoassay and indicated by the percent of untreated PAECs (control). The data are the mean $\pm \mathrm{SD}$ of 4 independent observations in separate cell culture wells. ${ }^{*} \mathrm{P}<0.05$ vs. control.

by multiple comparisons using Bonferroni's protected leastsignificant difference test. Statistical analysis was performed using Graph Pad Prism 5 (GraphPad Software, La Jolla, CA, USA). Data are presented as mean \pm SD and $\mathrm{P}<0.05$ considered statistically significant.

\section{Results}

Effects of FFAs on BK-Induced NO Production in PAECs

The effects of individual FFAs on endothelial function were elucidated by determining the effect of each FFA on NO pro- duction by fluorimetry with DAF-FM/DA. BK at $1 \mu \mathrm{mol} / \mathrm{L}$ increased the $\mathrm{F} 490 / \mathrm{F}_{0} 490$ DAF-FM ratio to $1.059 \pm 0.019$. The BK-induced increase in the F490/F0490 DAF-FM ratio was confirmed to be totally inhibited by pretreatment with $100 \mu \mathrm{mol} / \mathrm{L}$ of $L$-nitro-arginine methyl ester ( $L$-NAME), an endothelial NO synthase inhibitor (Figure 1A), which was consistent with our previous study's results. ${ }^{15} \mathrm{LA}$ reduced NO production by $62 \%$ and $89 \%$ at 0.1 and $1 \mu \mathrm{mol} / \mathrm{L}$, respectively; however, oleic, palmitic, or stearic acid at $10 \mu \mathrm{mol} / \mathrm{L}$ had no effect on BK-induced NO production in PAECs (Figure 1B). 
A

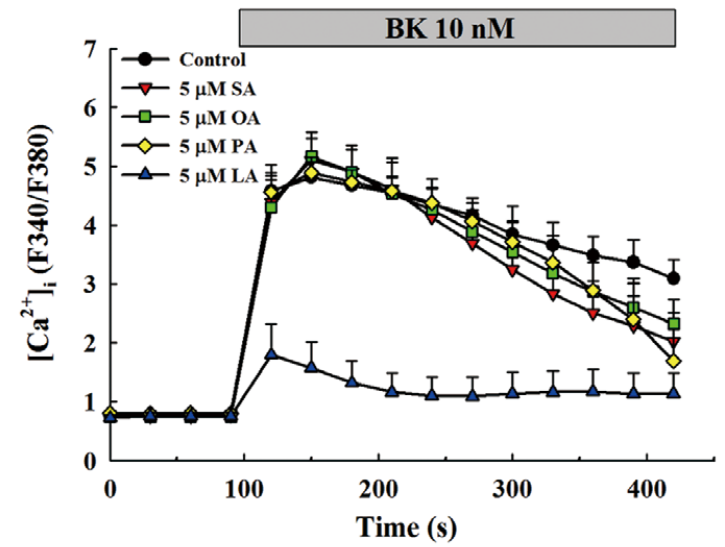

C

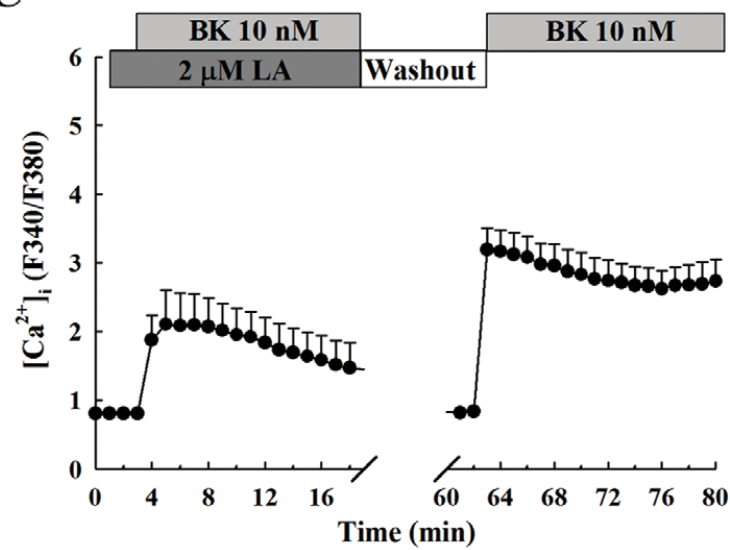

B

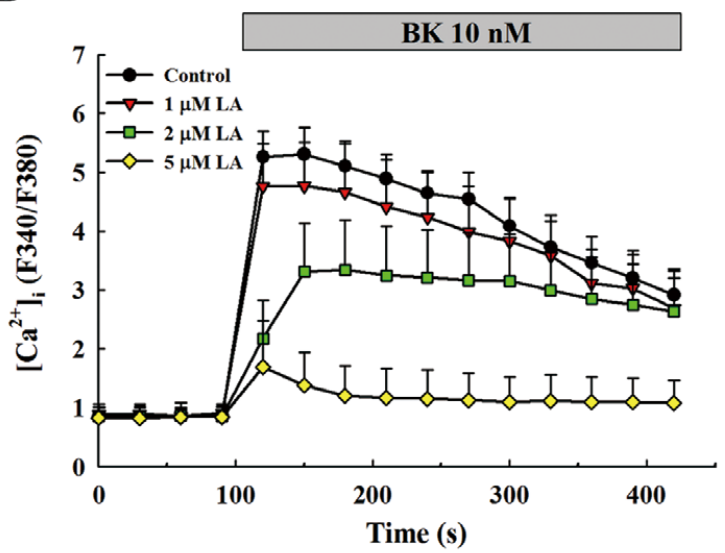

D

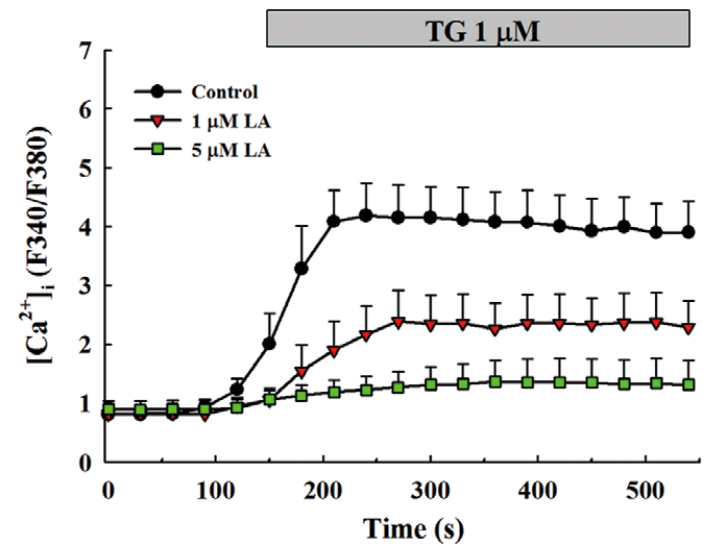

Figure 3. Effects of free fatty acids (FFAs) on endothelial $\mathrm{Ca}^{2+}$ responses in porcine aortic endothelial cells (PAECs). (A) Influence of FFAs on bradykinin (BK)-induced changes in intracellular $\mathrm{Ca}^{2+}$ concentration $\left(\left[\mathrm{Ca}^{2+}\right] \mathrm{i}\right)$. PAECs were pretreated with $5 \mu \mathrm{mol} / \mathrm{L}$ of linoleic (LA), palmitic (PA), stearic (SA), or oleic acid (OA) for $75 \mathrm{~min}$. (B) Effect of LA at various concentrations on BK-induced changes in $\left[\mathrm{Ca}^{2+}\right]_{i}$. (C) Reversible inhibitory effects of LA on BK-induced endothelial $\mathrm{Ca}^{2+}$ responses. After the inhibition of BKinduced $\mathrm{Ca}^{2+}$ responses caused by $2 \mu \mathrm{mol} / \mathrm{L}$ of $\mathrm{LA}$ was identified, BK and LA were washed out using a modified Tyrode's solution. BK was re-applied $40 \mathrm{~min}$ after the washout. (D) Effects of LA on thapsigargin (TG)-induced $\mathrm{Ca}^{2+}$ responses in PAECs. The data are the mean $\pm S D$ of 4 independent observations in separate cell culture wells.

\section{Effects of FFAs on BK-Induced PGI2 Production in PAECs}

The effect of FFAs on endothelial PGI2 production was determined by enzyme immunoassay of a stable PGI2 metabolite, 6-keto-PGF $1 \alpha$. BK at $10 \mathrm{nmol} / \mathrm{L}$ greatly increased the 6-keto-PGF $\alpha$ concentration from $116.1 \pm 14.9$ to $1519.9 \pm$ $117.8 \mathrm{fmol} / 10^{6}$ cells after $14 \mathrm{~min}$. Pretreatment with LA inhibited 6-keto-PGF $1 \alpha$ production by $30 \%, 51 \%$, and $71 \%$ at 1,5 , and $10 \mu \mathrm{mol} / \mathrm{L}$, respectively (Figure 2). Palmitic, oleic, or stearic acid had no effect on $\mathrm{BK}$-induced $\mathrm{PGI}_{2}$ production in PAECs.

\section{Effects of FFAs on Intracellular Ca ${ }^{2+}$ Responses in PAECs}

To verify whether the inhibitory effects of LA on EDRF production were caused by suppressing the intracellular $\mathrm{Ca}^{2+}$ responses that mediate EDRF production, ${ }^{15}$ the effects of FFAs on BK-induced $\mathrm{Ca}^{2+}$ responses in PAECs were tested. It was found that, in the presence of extracellular $\mathrm{Ca}^{2+}, 10 \mathrm{nmol} / \mathrm{L}$ of BK caused a rapid increase in the F340/F380 ratio of fura-2 from $0.77 \pm 0.07$ (basal) to $4.81 \pm 0.26$ (maximal), which then slowly attenuated (Figure 3A). Pretreatment with $5 \mu \mathrm{mol} / \mathrm{L}$ of
LA inhibited the BK-induced $\mathrm{Ca}^{2+}$ responses, but none of the other FFAs exhibited an effect (Figure 3A). Moreover, the intensity and duration of $\mathrm{BK}$-induced $\mathrm{Ca}^{2+}$ responses were reduced by LA in a dose-dependent manner (Figure 3B). The inhibition by $\mathrm{LA}$ of the $\mathrm{BK}$-induced $\mathrm{Ca}^{2+}$ responses was also observed in HUVECs (Figure S1A). To clarify whether this inhibitory effect of LA was reversible or not, the BK-induced $\mathrm{Ca}^{2+}$ responses after washout of LA by modified Tyrode's solution were examined. The removal of LA rejuvenated the BK-induced $\mathrm{Ca}^{2+}$ responses (Figure $3 \mathrm{C}$ ).

To evaluate which section of the endothelial $\mathrm{Ca}^{2+}$ signaling pathway was being affected by LA, the influence of LA on thapsigargin (TG)-induced $\mathrm{Ca}^{2+}$ responses was tested. TG, an irreversible inhibitor of endoplasmic reticulum (ER) $\mathrm{Ca}^{2+}$ ATPase, was used to investigate store-operated $\mathrm{Ca}^{2+}$ entry (SOCE). TG increases intracellular $\mathrm{Ca}^{2+}$ level through the plasma membrane store-operated $\mathrm{Ca}^{2+}$ channel (SOCC) activated by depleting ER $\mathrm{Ca}^{2+}$ stores. TG evoked a slightly delayed but long-lasting increase in F340/F380 from 0.80 0.11 (basal) to $4.19 \pm 0.55$ (maximal) in the presence of extracellular 
A

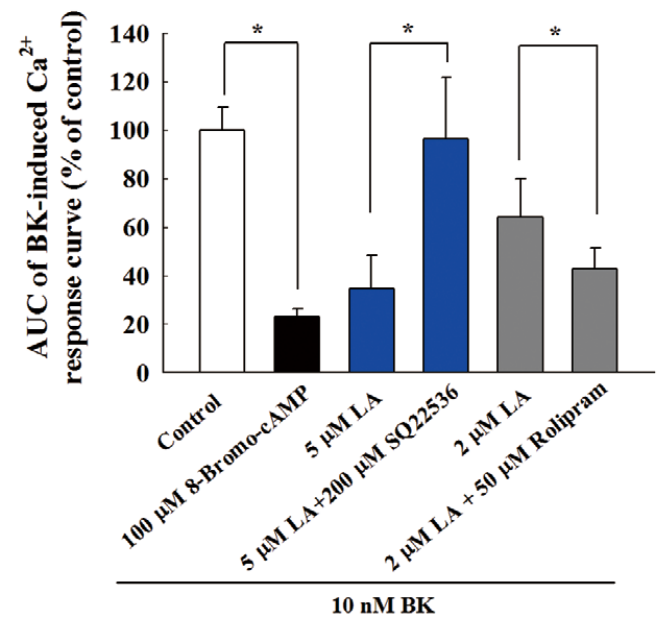

B

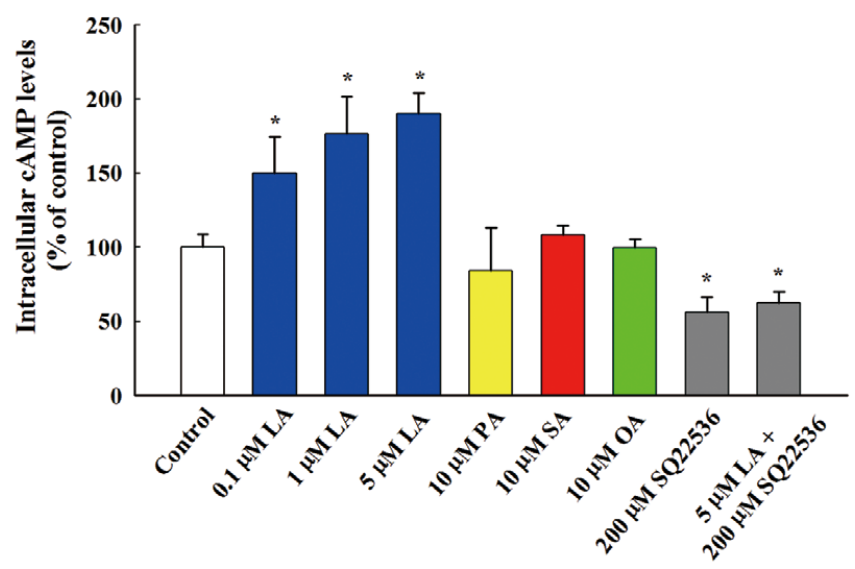

Figure 4. Involvement of intracellular cAMP formation in the inhibitory effect of linoleic acid (LA) on endothelial $\mathrm{Ca}^{2+}$ responses in porcine aortic endothelial cells (PAECs). (A) Influences of 8-bromo-cAMP, adenylate cyclase inhibitor and cAMP-hydrolyzing phosphodiesterase (PDE) inhibitor on the inhibitory effect of $\mathrm{LA}$ on endothelial $\mathrm{Ca}^{2+}$ responses induced by bradykinin (BK). PAECs were pretreated with 8-bromo-cAMP, LA, LA + SQ22563 (adenylate cyclase inhibitor),or LA+rolipram (cAMP-hydrolyzing PDE inhibitor) for $75 \mathrm{~min}$. The figure shows the percent of control (untreated ECs) in the area under the curve (AUC) of the endothelial $\mathrm{Ca}^{2+}$ responses from $90 \mathrm{~s}$, at BK administration, to $420 \mathrm{~s}$. Data are the mean \pm SD of 4 independent observations in separate cell culture wells. ${ }^{*} \mathrm{P}<0.05$. (B) Intracellular CAMP levels in PAECs treated with various free fatty acids (FFAs: palmitic (PA), stearic $(\mathrm{SA})$, oleic acid (OA)) and/or SQ22563. PAECs were incubated with the various FFAs and/or SQ22563 at the indicated concentrations for $75 \mathrm{~min}$. The intracellular cAMP level (open bar) in the PAECs incubated with a modified Tyrode's solution is indicated as the control. The data are the mean $\pm S D$ of 3 independent observations in separate cell culture wells. ${ }^{*} P<0.05$ vs. control.

A

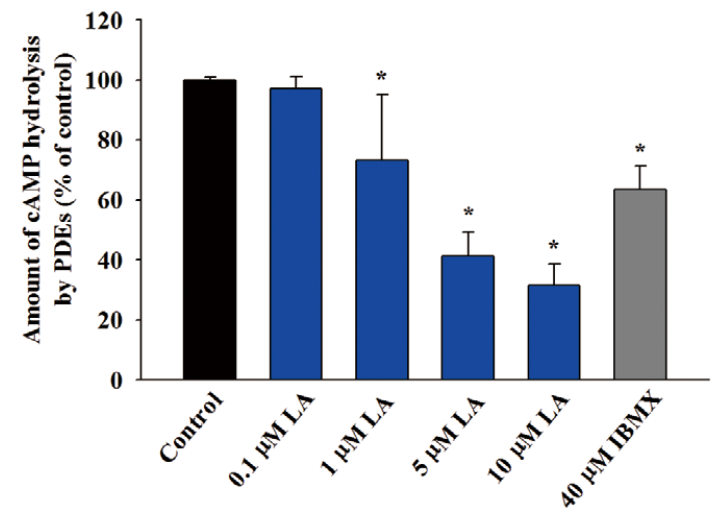

B

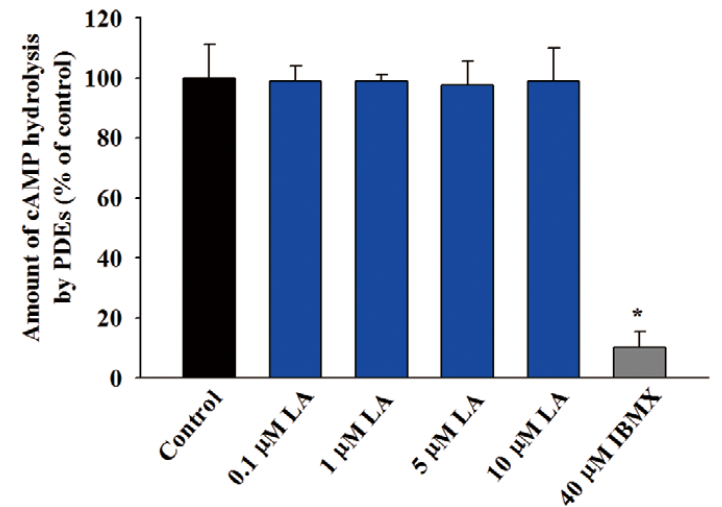

Figure 5. Effects of linoleic acid (LA) on cAMP/cGMP-hydrolyzing phosphodiesterase (PDE) activity in vitro. (A) cAMP-hydrolyzing PDE activity without (control) or with various concentrations of LA was evaluated as the cleavage of cAMP in $30 \mathrm{~min}$. IBMX, a nonspecific PDE inhibitor, was used as a test control. The summary indicates the percent of control in the amount of cAMP hydrolysis by cAMP-hydrolyzing PDE in vitro. (B) CGMP-hydrolyzing PDE activity in the presence or absence of LA. The data are the mean \pm SD of 6 independent observations in separate wells. ${ }^{*} \mathrm{P}<0.05$ vs. control.

$\mathrm{Ca}^{2+}$. Pretreatment with $1 \mu \mathrm{mol} / \mathrm{L}$ of LA obviously inhibited the TG-induced $\mathrm{Ca}^{2+}$ responses, and $5 \mu \mathrm{mol} / \mathrm{L}$ of LA almost abolished them (Figure 3D). The inhibition by LA of TG-induced $\mathrm{Ca}^{2+}$ responses was also observed in HUVECs (Figure S1B). These findings indicate that LA suppresses endothelial $\mathrm{Ca}^{2+}$ responses by inhibiting the SOCE signaling pathway between
$\mathrm{Ca}^{2+}$ depletion of ER $\mathrm{Ca}^{2+}$ stores and the SOCC.

\section{Involvement of Intracellular cAMP Formation in the Inhibitory} Effect of LA on Endothelial $\mathrm{Ca}^{2+}$ Responses in PAECs

The involvement of cAMP-related signaling pathways in the inhibition of endothelial $\mathrm{Ca}^{2+}$ responses by LA was explored 
because previous studies indicated that intracellular cAMP formation could be involved in the regulation of $\mathrm{Ca}^{2+}$ responses in ECs, ${ }^{16}$ and that intracellular cAMP levels was increased by LA in neuroblastoma cells..$^{17}$ In the present study, the inhibitory effect of LA on BK-induced endothelial $\mathrm{Ca}^{2+}$ responses was completely eliminated by an adenylate cyclase inhibitor, SQ22536, at $200 \mu \mathrm{mol} / \mathrm{L}$, and boosted by $50 \mu \mathrm{mol} / \mathrm{L}$ of rolipram, a selective cAMP-hydrolyzing PDE inhibitor (Figure 4A). A plasma membrane permeable cAMP, 8-bromo-cAMP, at $100 \mu \mathrm{mol} / \mathrm{L}$ suppressed the BK-induced $\mathrm{Ca}^{2+}$ responses (Figure 4A) and TG (data not shown). Furthermore, LA increased intracellular cAMP levels in a dose-dependent manner, and the intracellular cAMP formation induced by LA was abolished by SQ22536 (Figure 4B). Taken together, these findings strongly suggest that intracellular cAMP formation is involved in the inhibitory effects of LA on endothelial $\mathrm{Ca}^{2+}$ responses in PAECs.

\section{Involvement of cAMP-Hydrolyzing PDE Activity in Intracellular cAMP Formation Caused by LA}

To explore the mechanisms through which LA increases intracellular cAMP levels, we tested the effects of LA on cAMPhydrolyzing PDE activity in vitro. In a period of $30 \mathrm{~min}$, LA decreased the cleavage of cAMP through PDE by $27 \%, 59 \%$ and $68 \%$ at 1,5 and $10 \mu \mathrm{mol} / \mathrm{L}$, respectively, as measured by a PDE activity assay (Figure 5A). Other individual FFA at $10 \mu \mathrm{mol} / \mathrm{L}$ did not affect PDE activity (data not shown). A nonspecific PDE inhibitor, IBMX, was used as a test control and pharmacological inhibition of PDE by IBMX was confirmed (Figure 5A). These results suggest that LA directly suppresses cAMP-hydrolyzing PDE activity, which may be one of the mechanisms by which LA increases intracellular cAMP levels in PAECs. The effect of LA on cGMP-hydrolyzing PDE activity in vitro was also examined. We confirmed that LA did not show any inhibitory effects on cGMP-hydrolyzing PDE activity (Figure 5B).

\section{Discussion}

The present study aimed to investigate the effects of individual FFAs in human plasma on EDRF production, as well as the mechanisms by which the individual FFAs affected EDRF production. The results demonstrated that (1) among the various FFAs, only LA attenuated $\mathrm{NO}$ and $\mathrm{PGI}_{2}$ production in PAECs, (2) LA suppressed endothelial $\mathrm{Ca}^{2+}$ responses by intracellular cAMP accumulation via cAMP-hydrolyzing PDE inhibition, and (3) other FFAs, including palmitic, stearic and oleic acids, did not show any effects on EDRF production or endothelial $\mathrm{Ca}^{2+}$ responses.

An elevated plasma FFA level is associated with endothelial dysfunction and cardiovascular diseases. ${ }^{18-23}$ The Quebec Cardiovascular Study indicated that elevated plasma FFA concentrations are associated with an increased risk of developing symptomatic IHD (odds ratio 2.1, $\mathrm{P}=0.05$ ) independent of age, smoking habit, systolic blood pressure, low-density lipoprotein cholesterol level, and family history of IHD. ${ }^{1}$ Furthermore, in a subanalysis of the prospective randomized clinical trial JELIS (15,534 subjects), only a high concentration of LA was significantly associated with a higher risk of major coronary events (hazard ratio (HR) $1.33,95 \%$ confidence interval (CI) 1.02-1.74) in the control group of 7,722 subjects, and other FFAs, such as palmitic, stearic, oleic, arachidonic, docosahexaenoic, and eicosapentaenoic acids, did not exhibit any significant correlation with a risk of major coronary events. ${ }^{2}$ Most recently, evaluation of recovered data from the Sydney
Diet Heart Study, a single, blinded, parallel group, randomized controlled trial revealed that replacement of dietary saturated fats with LA resulted in increased rates of death from all causes, coronary artery disease, and cardiovascular disease. ${ }^{3}$ In the present study, of the major FFAs in human plasma, only LA was confirmed to attenuate EDRF production in PAECs; none of the other FFAs tested showed any inhibitory effects.

In the present study, LA inhibited BK-induced $\mathrm{NO}_{\text {and } \mathrm{PGI}_{2}}$ production by suppressing endothelial $\mathrm{Ca}^{2+}$ responses. Kim et al demonstrated that LA inhibited insulin-mediated NO production via Akt and eNOS phosphorylation, ${ }^{9}$ which was independent of intracellular $\mathrm{Ca}^{2+}$ responses. ${ }^{24}$ Thus, it is possible that LA suppresses NO production through 2 parallel pathways: the $\mathrm{Ca}^{2+}$ response-mediated $\mathrm{NO}$ production and that via Akt and eNOS phosphorylation. On the other hand, Saraswathi et al reported that $3 \mathrm{~h}$ incubation of LA at $90 \mu \mathrm{mol} / \mathrm{L}$ increased the intracellular $\mathrm{Ca}^{2+}$ concentration and $\mathrm{Ca}^{2+}$-dependent $\mathrm{NO}$ production in porcine pulmonary arterial ECs. ${ }^{10}$ However, we found that $\mathrm{LA}$ at $0.1-10 \mu \mathrm{mol} / \mathrm{L}$ did not affect the baseline intracellular $\mathrm{Ca}^{2+}$ concentration (data not shown), but did attenuate the $\mathrm{BK}$-induced $\mathrm{Ca}^{2+}$ responses and $\mathrm{NO}$ production in PAECs (Figures 1B,3A,3B). Our present results, together with those from Saraswathi et al study, suggest that the effect of LA on $\mathrm{Ca}^{2+}$-dependent NO production in ECs may have 2 aspects: LA at low doses (approximately $0.1-10 \mu \mathrm{mol} / \mathrm{L}$ ) attenuates the $\mathrm{Ca}^{2+}$ response-mediated NO production stimulated by an agonist such as BK, and LA at higher doses is able to raise intracellular $\mathrm{Ca}^{2+}$ levels and therefore increases $\mathrm{Ca}^{2+}$ dependent NO production, which transforms into peroxynitrite reacting with superoxide. ${ }^{10} \mathrm{LA}$ at both of dosages could disturb vascular homeostasis.

It was recently reported that a 3-day incubation of human aortic ECs with LA increased monocyte chemotaxis and adhesion to ECs, which may exert proinflammatory and proatherogenic effects. ${ }^{5}$ EDRFs such as $\mathrm{NO}$ and $\mathrm{PGI}_{2}$ are known to prevent the abnormal constriction of coronary arteries, inhibit the aggregation of platelets, and suppress the chemotaxis, adhesion and penetration of monocyte to vascular endothelium, ${ }^{25,26}$ thus sustained attenuation of EDRF production, such as results from the exposure of ECs to an high level of LA, may not only be responsible for the promotion of monocyte chemotaxis and adhesion, but also contribute to the multistep process of atherosclerogenesis.

EDRFs are defined as vasodilators that are synthesized and released by endothelium, and include not only PGI 2 and NO, but also endothelium-derived hyperpolarizing factors (EDHF) such as $\mathrm{H}_{2} \mathrm{O}_{2}$ and epoxyeicosatrienoic acids. EDHF-mediated vasodilatation is essential for maintaining of cardiovascular homeostasis, especially in microvessels. ${ }^{27,28}$ EDHF-mediated vasodilatation is relatively resistant to atherosclerosis and therefore capable of serving as a back-up system for suppressed NO-mediated vasodilatation. The mechanisms of enhanced EDHF-mediated vasodilatation have been well illustrated. ${ }^{29}$ The regulation of endothelium function varies according to the site and the type of blood vessel. ${ }^{30}$ The contribution of each individual EDRF to endothelium-dependent relaxation differs between the aorta and the small vessels; for example, NO plays a prominent role in aorta, but EDHF is prominent in the mesenteric artery. ${ }^{31}$ Our previous study showed that the EDHFmediated arterial hyperpolarization and relaxation also depends on endothelial $\mathrm{Ca}^{2+}$ responses. ${ }^{32}$ Because of the inhibitory effects on endothelial $\mathrm{Ca}^{2+}$ responses exhibited by LA in the present study, LA may also interfere in EDHF-mediated vascular responses in the small blood vessels.

Regarding the mechanism by which LA attenuates EDRF 
production, we verified that LA inhibits the endothelial $\mathrm{Ca}^{2+}$ responses that regulate EDRF production. LA was also confirmed to inhibit TG-induced $\mathrm{Ca}^{2+}$ responses, which suggests that it inhibits the signaling pathways of SOCE between $\mathrm{Ca}^{2+}$ depletion of the $\mathrm{ER} \mathrm{Ca}^{2+}$ stores and the plasma membrane $\mathrm{Ca}^{2+}$-permeable cation channels activated by $\mathrm{Ca}^{2+}$ depletion of ER Ca ${ }^{2+}$ stores. ${ }^{33,34}$

Luckhoff et al reported that a PGI2 analog (iloprost) and $\beta$-adrenergic stimulant (isoproterenol) inhibited endothelial $\mathrm{Ca}^{2+}$ responses by intracellular cAMP formation and deduced that enhanced turnover of cAMP in ECs may protect the organism from sudden, overwhelming release of powerful EDRFs. ${ }^{16}$ Thus, in the present study, the possible involvement of cAMPrelated signaling pathways in the inhibitory effect of LA on endothelial $\mathrm{Ca}^{2+}$ responses was examined. Our results showed that (1) LA increased intracellular cAMP levels, (2) the inhibitory effect of LA on endothelial $\mathrm{Ca}^{2+}$ responses was eliminated by an adenylate cyclase inhibitor, SQ22563, boosted by a PDE4 inhibitor, rolipram, and mimicked by 8-bromo-cAMP, and (3) LA selectively inhibited cAMP-hydrolyzing PDE activity in vitro. These findings in PAECs suggest that LA suppresses endothelial $\mathrm{Ca}^{2+}$ responses by intracellular cAMP formation via cAMP-hydrolyzing PDE inhibition.

The intracellular cAMP concentration results from the balance between its production by adenylate cyclase and its metabolism by cAMP-hydrolyzing PDE. The present results show that LA suppresses cAMP-hydrolyzing PDE activity, which at least in part contributed to the accumulation of intracellular cAMP in PAECs. As shown in Figure 4B, SQ22563 significantly reduced the baseline intracellular cAMP level, which indicates that in the steady state without any stimulants, cAMP is constantly produced via adenylate cyclase. Therefore, suppression of cAMP-hydrolyzing PDE activity by LA could result in an increase in the intracellular cAMP level, even in the steady state, in ECs. Interestingly, LA exhibited a selective inhibitory effect only on cAMP-hydrolyzing PDE, but not on cGMP-hydrolyzing PDE. This selectivity of inhibition by LA on PDEs was demonstrated in vitro for the first time in the present study. The other FFAs used in our study did not show any inhibitory effects on cAMP-hydrolyzing PDE activity.

\section{Study Limitations}

There are some limitations of the present study worth mentioning. First, EDRF-mediated modulation of vascular tone varies on the type of blood vessel. The data in our study were only obtained from aortic ECs, so whether LA suppresses EDRF production in ECs from smaller arteries, such as the coronary and mesenteric arteries, remains to be verified. Second, we only showed that LA suppressed NO and $\mathrm{PGI}_{2}$ production; however, the effects of LA on other EDRFs, such as EDHF, should be further elucidated. Third, our findings should be confirmed in an EDRF-mediated relaxation model, such as the endothelium-dependent relaxation of aorta.

\section{Conclusions}

The present study demonstrated that LA induced intracellular cAMP accumulation by selectively inhibiting cAMP-hydrolyzing PDE activity, and thereby attenuating endothelial $\mathrm{Ca}^{2+}$ responses and EDRF production in PAECs.

\section{Acknowledgments}

This study was supported in part by Grants-in-Aid (22590499) for Scientific Research from the Ministry of Education, Culture, Sports, Science and Technology of Japan to K. Takeuchi. We thank Ms. Michiko Kageyama for her help in preparing the porcine endothelial cells.

\section{Disclosures}

The authors declare no conflicts of interest.

\section{References}

1. Pirro M, Mauriege P, Tchernof A, Cantin B, Dagenais GR, Despres $\mathrm{JP}$, et al. Plasma free fatty acid levels and the risk of ischemic heart disease in men: Prospective results from the Quebec Cardiovascular Study. Atherosclerosis 2002; 160: 377-384.

2. Itakura H, Yokoyama M, Matsuzaki M, Saito Y, Origasa H, Ishikawa $\mathrm{Y}$, et al. Relationships between plasma fatty acid composition and coronary artery disease. J Atheroscler Thromb 2011; 18: 99-107.

3. Ramsden CE, Zamora D, Leelarthaepin B, Majchrzak-Hong SF, Faurot KR, Suchindran CM, et al. Use of dietary linoleic acid for secondary prevention of coronary heart disease and death: Evaluation of recovered data from the Sydney Diet Heart Study and updated meta-analysis. BMJ 2013; 346: e8707.

4. Fei J, Cook C, Gillespie M, Yu B, Fullen K, Santanam N. Atherogenic omega-6 lipids modulate PPAR-EGR-1 crosstalk in vascular cells. PPAR Res 2011 October 26, doi:10.1155/2011/753917 [E-pub ahead of print].

5. Matesanz N, Jewhurst V, Trimble ER, McGinty A, Owens D, Tomkin $\mathrm{GH}$, et al. Linoleic acid increases monocyte chemotaxis and adhesion to human aortic endothelial cells through protein kinase $\mathrm{C}$ - and cyclooxygenase-2-dependent mechanisms. J Nutr Biochem 2012; 23: 685-690.

6. Takahashi M, Ikeda U, Masuyama J, Funayama H, Kano S, Shimada K. Nitric oxide attenuates adhesion molecule expression in human endothelial cells. Cytokine 1996; 8: 817-821.

7. Braun M, Pietsch P, Zepp A, Schror K, Baumann G, Felix SB. Regulation of tumor necrosis factor alpha- and interleukin-1-betainduced induced adhesion molecule expression in human vascular smooth muscle cells by cAMP. Arterioscler Thromb Vasc Biol 1997; 17: $2568-2575$.

8. Tsao PS, Wang B, Buitrago R, Shyy JY, Cooke JP. Nitric oxide regulates monocyte chemotactic protein-1. Circulation 1997; 96: 934-940.

9. Kim F, Tysseling KA, Rice J, Pham M, Haji L, Gallis BM, et al. Free fatty acid impairment of nitric oxide production in endothelial cells is mediated by IKKbeta. Arterioscler Thromb Vasc Biol 2005; 25: 989-994.

10. Saraswathi V, Wu G, Toborek M, Hennig B. Linoleic acid-induced endothelial activation: Role of calcium and peroxynitrite signaling. J Lipid Res 2004; 45: 794-804.

11. Miwa H, Yamamoto M, Nishida T, Nunoi K, Kikuchi M. Highperformance liquid chromatographic analysis of serum long-chain fatty acids by direct derivatization method. J Chromatogr 1987; 416: $237-245$.

12. Watanabe H, Takahashi R, Zhang XX, Kakizawa H, Hayashi H, Ohno R. Inhibition of agonist-induced $\mathrm{Ca}^{2+}$ entry in endothelial cells by myosin light-chain kinase inhibitor. Biochem Biophys Res Commun 1996; 225: $777-784$.

13. Kojima H, Nakatsubo N, Kikuchi K, Kawahara S, Kirino Y, Nagoshi $\mathrm{H}$, et al. Detection and imaging of nitric oxide with novel fluorescent indicators: Diaminofluoresceins. Anal Chem 1998; 70: 2446-2453.

14. Kaw S, Hecker M. Endothelium-derived hyperpolarizing factor, but not nitric oxide or prostacyclin release, is resistant to menadione-induced oxidative stress in the bovine coronary artery. Naunyn Schmiedebergs Arch Pharmacol 1999; 359: 133-139.

15. Asai M, Takeuchi K, Saotome M, Urushida T, Katoh H, Satoh H, et al. Extracellular acidosis suppresses endothelial function by inhibiting store-operated $\mathrm{Ca}^{2+}$ entry via non-selective cation channels. Cardiovasc Res 2009; 83: 97-105.

16. Luckhoff A, Mulsch A, Busse R. cAMP attenuates autacoid release from endothelial cells: Relation to internal calcium. Am J Physiol 1990; 258: H960-H966.

17. Murphy MG, Byczko Z. Further studies of the mechanism(s) of polyunsaturated-fatty-acid-mediated increases in intracellular cAMP formation in N1E-115 neuroblastoma cells. Neurochem Res 1992; 17: $1069-1077$.

18. Reaven GM, Hollenbeck C, Jeng CY, Wu MS, Chen YD. Measurement of plasma glucose, free fatty acid, lactate, and insulin for $24 \mathrm{~h}$ in patients with NIDDM. Diabetes 1988; 37: 1020-1024.

19. Muller JE, Tofler GH, Stone PH. Circadian variation and triggers of onset of acute cardiovascular disease. Circulation 1989; 79: 733 743.

20. Steinberg HO, Chaker H, Leaming R, Johnson A, Brechtel G, Baron 
$\mathrm{AD}$. Obesity/insulin resistance is associated with endothelial dysfunction: Implications for the syndrome of insulin resistance. $J$ Clin Invest 1996; 97: 2601-2610.

21. Carlsson M, Wessman Y, Almgren P, Groop L. High levels of nonesterified fatty acids are associated with increased familial risk of cardiovascular disease. Arterioscler Thromb Vasc Biol 2000; 20: $1588-1594$.

22. Kocer A, Ilhan A, Ince N, Bilge C. The related causes in very early morning onset of stroke. Prog Neuropsychopharmacol Biol Psychiatry 2005; 29: 983-988.

23. Pilz S, Scharnagl H, Tiran B, Seelhorst U, Wellnitz B, Boehm BO, et al. Free fatty acids are independently associated with all-cause and cardiovascular mortality in subjects with coronary artery disease. $J$ Clin Endocrinol Metab 2006; 91: 2542-2547.

24. Takahashi S, Mendelsohn ME. Synergistic activation of endothelial nitric-oxide synthase (eNOS) by HSP90 and Akt: Calcium-independent eNOS activation involves formation of an HSP90-Akt-CaMbound eNOS complex. J Biol Chem 2003; 278: 30821-30827.

25. Vanhoutte PM. Endothelial dysfunction: The first step toward coronary arteriosclerosis. Circ J 2009; 73: 595-601.

26. Kawabe J, Ushikubi F, Hasebe N. Prostacyclin in vascular diseases: Recent insights and future perspectives. Circ J 2010; 74: 836-843.

27. Urakami-Harasawa L, Shimokawa H, Nakashima M, Egashira K, Takeshita A. Importance of endothelium-derived hyperpolarizing factor in human arteries. J Clin Invest 1997; 100: 2793-2799.

28. Shimokawa H. Hydrogen peroxide as an endothelium-derived hyperpolarizing factor. Pflugers Arch 2010; 459: 915-922.

29. Ohashi J, Sawada A, Nakajima S, Noda K, Takaki A, Shimokawa H. Mechanisms for enhanced endothelium-derived hyperpolarizing factor-mediated responses in microvessels in mice. Circ J 2012; 76:
$1768-1779$.

30. Heo KS, Fujiwara K, Abe J. Disturbed-flow-mediated vascular reactive oxygen species induce endothelial dysfunction. Circ J 2011; 75: $2722-2730$.

31. Shimokawa H, Yasutake H, Fujii K, Owada MK, Nakaike R, Fukumoto Y, et al. The importance of the hyperpolarizing mechanism increases as the vessel size decreases in endothelium-dependent relaxations in rat mesenteric circulation. $J$ Cardiovasc Pharmacol 1996; 28: 703-711.

32. Fukao M, Watanabe H, Takeuchi K, Tomioka H, Hattori Y. Effects of SK\&F 96365 and mefenamic acid on $\mathrm{Ca}^{2+}$ influx in stimulated endothelial cells and on endothelium-derived hyperpolarizing factormediated arterial hyperpolarization and relaxation. J Cardiovasc Pharmacol 2001; 38: 130-140.

33. Takeuchi K, Watanabe H, Tran QK, Ozeki M, Uehara A, Katoh H, et al. Effects of cytochrome P450 inhibitors on agonist-induced $\mathrm{Ca}^{2+}$ responses and production of $\mathrm{NO}$ and $\mathrm{PGI}_{2}$ in vascular endothelial cells. Mol Cell Biochem 2003; 248: 129-134.

34. Tran QK, Ohashi K, Watanabe H. Calcium signalling in endothelial cells. Cardiovasc Res 2000; 48: 13-22.

\section{Supplementary Files}

Supplementary File 1

Figure S1. Effects of linoleic acid (LA) on endothelial $\mathrm{Ca}^{2+}$ responses in human umbilical vein endothelial cells (HUVECs).

Please find supplementary file(s);

http://dx.doi.org/10.1253/circj.CJ-13-0248 